\title{
STRANGE EXPECTATIONS AND THE WINNIE-THE-POOH PROBLEM
}

\author{
MARKO THIEL AND NATHAN WILLIAMS
}

\begin{abstract}
Motivated by the study of simultaneous cores, we give three proofs (in varying levels of generality) that the expected norm of a weight in a highest weight representation $V_{\lambda}$ of a complex simple Lie algebra $\mathfrak{g}$ is $\frac{1}{h+1}(\lambda+2 \rho, \lambda)$. First, we argue directly using the polynomial method and the Weyl character formula. Second, we use the combinatorics of semistandard tableaux to obtain the result in type $A$. Third, and most interestingly, we relate this problem to the "Winnie-the-Pooh problem" regarding orthogonal decompositions of Lie algebras; although this approach offers the most explanatory power, it applies only to Cartan types other than $A$ and $C$. We conclude with computations of many combinatorial cumulants.
\end{abstract}

\section{INTRODUCTION}

The representation theory of complex simple Lie algebras is a classical source of algebraic combinatorics, intimately related to tableaux and plane partitions, symmetric functions and positivity questions, quantum groups and crystals, and the plactic monoid and RSK.

Having fixed a Cartan subalgebra $\mathfrak{h}$, the finite-dimensional irreducible representions of a complex simple Lie algebra $\mathfrak{g}$ are completely classified by the dominant weights $\lambda$ in its weight lattice $\Lambda \subset \mathfrak{h}^{*}$. Using the symmetric bilinear form $\langle\cdot, \cdot\rangle$ on $\mathfrak{h}^{*}$ induced by the Killing form and writing $\rho$ for the half-sum of the positive roots $\Phi^{+}$, the Weyl dimension formula asserts that for $\lambda$ dominant, the dimension of the finite-dimensional irreducible representation $V_{\lambda}$

$$
\operatorname{dim}\left(V_{\lambda}\right)=\prod_{\alpha \in \Phi^{+}} \frac{\langle\alpha, \lambda+\rho\rangle}{\langle\alpha, \rho\rangle} .
$$

Motivated by the recent interest in statistics on simultaneous cores (see Section 3), the purpose of this paper is to give a formula for the average norm of a weight in a highest weight representation.

Theorem 1.1. For $\mathfrak{g}$ a complex simple Lie algebra with $V_{\lambda}$ its finite-dimensional irreducible representation of highest weight $\lambda$, the expected norm of a weight in $V_{\lambda}$ is

$$
\underset{\mu \in V_{\lambda}}{\mathbb{E}}(\langle\mu, \mu\rangle)=\frac{1}{\operatorname{dim}\left(V_{\lambda}\right)} \sum_{\mu \in V_{\lambda}} \operatorname{dim}\left(V_{\lambda}(\mu)\right)\langle\mu, \mu\rangle=\frac{1}{h+1}\langle\lambda, \lambda+2 \rho\rangle,
$$

where $\operatorname{dim}\left(V_{\lambda}(\mu)\right)$ is the multiplicity of $\mu$ in $V_{\lambda}$ and $h$ is the Coxeter number of $\mathfrak{g}$.

Theorem 1.1 is illustrated in Figures 1 and 2 for highest weight representations for $\mathfrak{s l}_{3}$ and for $\mathfrak{s p}_{4}$.

Date: November 7, 2018.

2000 Mathematics Subject Classification. Primary 05E45; Secondary 20F55, 13 F60.

Key words and phrases. Lie algebra, highest weight representation, weight lattice, expected value. 


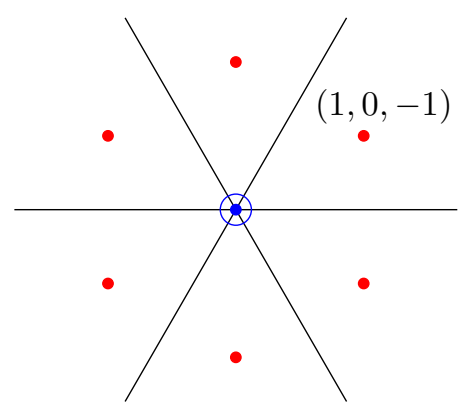

Figure 1 . In $\mathfrak{s l}_{3}$, the eight weights in $V_{\lambda}$ with $\lambda=(1,0,-1)$. The average norm is $\frac{6 \cdot 2+2 \cdot 0}{8}=\frac{3}{2}=\frac{1}{3+1}\langle\lambda, \lambda+2 \rho\rangle$.

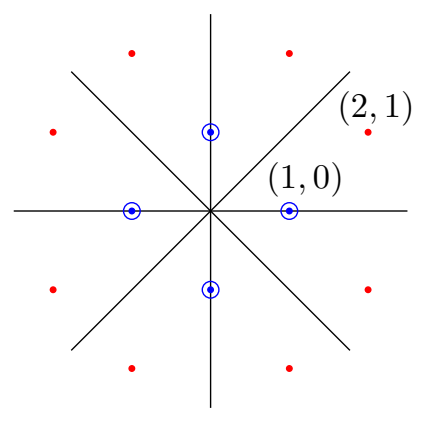

Figure 2 . In $\mathfrak{s p}_{4}$, the sixteen weights in $V_{\lambda}$ with $\lambda=(2,1)$. The average norm is $\frac{8 \cdot 5+4 \cdot 2 \cdot 1}{16}=3=\frac{1}{4+1}\langle\lambda, \lambda+2 \rho\rangle$.

\section{Lie Algebras and their Representation Theory}

Recall that the complex simple Lie algebras are classified by their Dynkin diagrams, illustrated in Figure 3. Fix a complex simple Lie algebra $\mathfrak{g}$ with Cartan subalgebra $\mathfrak{h}$; all Cartan subalgebras of $\mathfrak{g}$ are conjugate. Given a complex representation $V: \mathfrak{g} \rightarrow \mathfrak{g l}(V)$, we say that the weight space for $\mu \in \mathfrak{h}^{*}$ is the subspace

$$
V(\mu)=\{v \in V: H \cdot v=\mu(H) v \text { for all } H \in \mathfrak{h}\} .
$$

The adjoint representation of $\mathfrak{g}$ has non-zero weights called roots, and we obtain the Cartan decomposition

$$
\mathfrak{g}=\mathfrak{h} \oplus \bigoplus_{\alpha \in \Phi^{+}} \mathfrak{g}_{\alpha} \oplus \bigoplus_{\alpha \in \Phi^{-}} \mathfrak{g}_{\alpha}
$$

where $\Phi^{+}$and $\Phi^{-}$are the positive and negative roots, respectively. A simple root is a positive root that cannot be written as the sum of two positive roots, and we write $\widetilde{\alpha}$ for the highest root.

The Killing form is the nondegenerate symmetric bilinear form defined by

$$
B(X, Y)=\operatorname{tr}(\operatorname{ad}(X), \operatorname{ad}(Y)) .
$$

We normalize the Killing form so that the norm of a long root is 2, and we will write this normalized form as $\langle\cdot, \cdot\rangle$. We write $\|\alpha\|^{2}:=\langle\alpha, \alpha\rangle$.

Restricting the Killing form to $\mathfrak{h}$ and writing $\operatorname{dim}(\mathfrak{h})=n$ allows us to view weights and roots as points in $\mathbb{R}^{n}$, and the Weyl group of $\mathfrak{g}$ is the reflection group $W$ generated by the reflections perpendicular to the roots $\alpha \in \Phi$. The coroot of a $\operatorname{root} \alpha$ is $\alpha^{\vee}:=\frac{2 \alpha}{\langle\alpha, \alpha\rangle}$. 

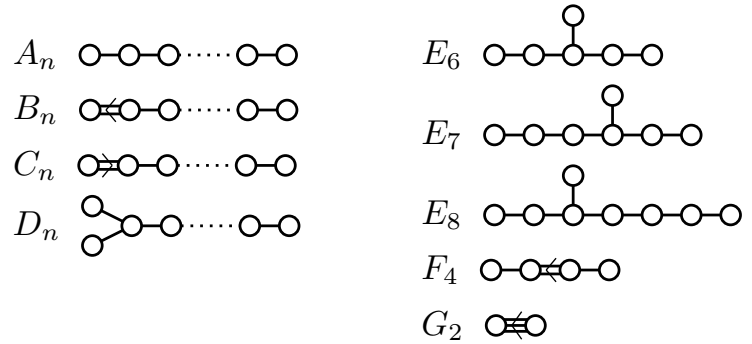

Figure 3. The Dynkin diagrams.

Let $\mathcal{A}\left[\mathfrak{h}^{*}\right]$ be the character ring of formal linear combinations of formal exponentials of weights. For a weight $\lambda \in \mathfrak{h}^{*}$, let

$$
A_{\lambda}=\sum_{w \in W} \operatorname{sgn}(w) e^{w(\lambda)} \in \mathcal{A}\left[\mathfrak{h}^{*}\right]
$$

Then $A_{\lambda}$ is alternating with respect to the $W$-action on $\mathcal{A}\left[\mathfrak{h}^{*}\right]$, so that $w\left(A_{\lambda}\right)=$ $\operatorname{sgn}(w) A_{\lambda}$, and it is also a $W$-alternating function of $\lambda: A_{w(\lambda)}=\operatorname{sgn}(w) A_{\lambda}$.

A weight $\lambda$ is called regular if $w(\lambda) \neq \lambda$ for all $e \neq w \in W$-in particular, $A_{\lambda}=0$ if $\lambda$ is not regular. It is called integral if its inner product with every coroot is integral. The fundamental weights are the dual basis to the simple coroots, and a weight is called dominant if it is a nonnegative linear combination of the fundamental weights. Let $\rho$ denote the lowest regular dominant integral weight.

Theorem 2.1 (Weyl character formula). If $\lambda$ is an integral weight, there exists a unique $f_{\lambda} \in \mathcal{A}\left[\mathfrak{h}^{*}\right]$ with

$$
A_{\lambda}=f_{\lambda} A_{\rho} .
$$

Furthermore, if $\lambda$ is dominant, then

$$
f_{\lambda+\rho}=\sum_{\mu \in \mathfrak{h}^{*}} \operatorname{dim}\left(V_{\lambda}(\mu)\right) e^{\mu}
$$

is the character of the finite dimensional irreducible representation $V_{\lambda}$, where the multiplicity of $\mu$ in $V_{\lambda}$ is denoted $\operatorname{dim}\left(V_{\lambda}(\mu)\right)$.

2.1. Casimir Elements and the Universal Enveloping Algebra. The HarishChandra isomorphism is an isomorphism between the center of the universal enveloping algebra of $\mathfrak{g}, Z(U(\mathfrak{g}))$, and $W$-invariant polynomials $S(\mathfrak{h})^{W}$. By the Shephard-Todd-Chevelley theorem, $S(\mathfrak{h})^{W}$ is a polynomial algebra with $n$ generators, and the degrees $d_{1}, d_{2}, \ldots, d_{n}$ of these generators play an important numerological role: for example, the highest degree is the Coxeter number $h$ (the order of a Coxeter element of $W)$, the dimension of the Lie algebra is $\operatorname{dim}(\mathfrak{g})=n(h+1)$, the number of reflections in $W$ is $\sum_{i=1}^{n}\left(d_{i}-1\right)$, and the number of elements in $W$ is $|W|=\prod_{i=1}^{n} d_{i}$. See also Section 7 for further numerology.

We call an element of $Z(U(\mathfrak{g}))$ a Casimir element - the Harish-Chandra isomorphism combined with the Shephard-Todd-Chevelley theorem shows that there are $n$ algebraically independent Casimir elements. Special emphasis is given to the Casimir element of degree two, which may be defined as follows: fixing any basis $\left\{X_{i}\right\}_{i=1}^{\operatorname{dim}(\mathfrak{g})}$, we obtain a dual basis $\left\{X^{i}\right\}_{i=1}^{\operatorname{dim}(\mathfrak{g})}$ using the Killing form, and then define

$$
\Omega=\sum_{i=1}^{\operatorname{dim}(\mathfrak{g})} X_{i} X^{i} \in Z(U(\mathfrak{g})) .
$$


As representations of $\mathfrak{g}$ coincide with modules for its universal enveloping algebra, Schur's lemma implies that since $\Omega$ is in the center of $U(\mathfrak{g})$, it acts as a scalar on any highest weight representation of $\mathfrak{g}$. The following well-known theorem explicitly identifies this scalar.

Theorem 2.2. Let $\lambda$ be a dominant weight. Then $\Omega$ acts as multiplication by $\langle\lambda, \lambda+2 \rho\rangle$ on $V_{\lambda}$.

Proof. Using the Cartan decomposition of Equation (2), write $\Omega=\sum_{1<i<n} H_{i} H_{i}^{*}+$ $\sum_{\alpha \in \Phi} E_{\alpha} E_{-\alpha}$, where we have chosen $\left\langle E_{\alpha}, E_{-\alpha}\right\rangle=1$ and $\left[E_{\alpha}, E_{-\alpha}\right]=\bar{H}_{\alpha}$. In the representation $V_{\lambda}$, we compute

$$
\begin{aligned}
\Omega & =\sum_{1 \leq i \leq n} H_{i} H_{i}^{*}+\sum_{\alpha \in \Phi} E_{\alpha} E_{-\alpha}=\sum_{1 \leq i \leq n} H_{i} H_{i}^{*}+2 \sum_{\alpha \in \Phi^{+}} E_{-\alpha} E_{\alpha}+\sum_{\alpha \in \Phi^{+}}\left[E_{\alpha}, E_{-\alpha}\right] \\
& =\sum_{1 \leq i \leq n} H_{i} H_{i}^{*}+2 \sum_{\alpha \in \Phi^{+}} E_{-\alpha} E_{\alpha}+\sum_{\alpha \in \Phi^{+}} H_{\alpha} .
\end{aligned}
$$

Acting on a highest weight vector in $V_{\lambda}$, the term $2 \sum_{\alpha \in \Phi^{+}} E_{-\alpha} E_{\alpha}$ vanishes, leaving only $\sum_{1 \leq i \leq n} \lambda\left(H_{i}\right) \lambda\left(H_{i}^{*}\right)+\lambda\left(\sum_{\alpha \in \Phi^{+}} H_{\alpha}\right)$. The first term is now computed as $\langle\lambda, \lambda\rangle$, while the second term gives $\langle\lambda, 2 \rho\rangle$ for $\rho$ the half sum of the positive roots.

\section{Motivation: Cores And Ehrhart Theory}

In this section we relate a special case of Theorem 1.1 (for the first fundamantal weight in type $A$ ) to the study of simultaneous core partitions.

3.1. Simultaneous Cores and Armstrong's Conjecture. An $a$-core is an integer partition with no hook-length of size $a$. The study of simultaneous $(a, b)$-coresthat is, partitions that are both $a$-cores and $b$-cores - is a topic that has recently seen quite a lot of interest from the combinatorics community [SZ15, Agg15]. When $\operatorname{gcd}(a, b)=1$, Anderson proved that the number of $(a, b)$-cores has the simple expression

$$
|\operatorname{core}(a, b)|=\frac{1}{a+b}\left(\begin{array}{c}
a+b \\
b
\end{array}\right)
$$

by giving a bijection to Dyck paths in an $a \times b$ rectangle [And02]. It is well-known that the dominant alcoves in the affine symmetric group $\widetilde{S}_{a}$ are naturally indexed by $a$-cores, and in this language Anderson's result had previously been proven in the generality of affine Weyl groups by both Haiman and Suter [Hai94, Sut98b].

While investigating the interpretation of $q, t$-statistics and the zeta map using the affine symmetric group [Arm15, AHJ14], Armstrong was led to conjecture that the expected number of boxes of a simultaneous core (its "size") had a beautiful formula.

Theorem 3.1 (Amstrong (conjectured), Johnson (proof) [Joh15]). The expected number of boxes of a simultaneous core is given by

$$
\underset{\lambda \in \operatorname{core}(a, b)}{\mathbb{E}}(\operatorname{size}(\lambda))=\frac{(a-1)(b-1)(a+b+1)}{24} .
$$

Example 3.2. We compute the expected number of boxes for the five simultaneous $(3,4)$-cores

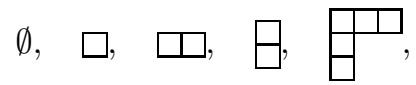

as

$$
\frac{1}{5}(0+1+2+2+5)=2=\frac{(3-1)(4-1)(3+4+1)}{24} .
$$


Theorem 3.1 was first proven by Johnson using Ehrhart theory [Joh15]. Building on Johnson's approach in [TW17], we showed that the statistic size could be interpreted as a slight modification of the natural norm on the weight space (see Figure 4), and generalized the result to all simply-laced affine Weyl groups (we now have a generalization to all affine Weyl groups):

$$
\operatorname{size}_{b}(x):=\frac{h}{2}\left\|x-b \frac{\rho^{\vee}}{h}\right\|^{2}-\frac{h}{2}\left\|\frac{\rho^{\vee}}{h}\right\|^{2} .
$$

Briefly, by composing the bijection between $a$-cores and dominant alcoves in $\widetilde{S}_{a}$, and the natural bijection between dominant alcoves and coroots, one obtains a bijection between simultaneous $(a, b)$-cores and $Q^{\vee} \cap b \mathcal{A}$ - coroot points inside a $b$-fold dilation of the fundamental alcove in $\widetilde{S}_{a}$. When $a$ is coprime to $b$, the cyclic symmetry of the affine Dynkin diagram gives rise to an affine isometry that partitions the weights inside $b \mathcal{A}$ into regular orbits, each of which contains a single coroot. It is therefore enough to consider the coweights in the dilation of the fundamental alcove $\Lambda^{\vee} \cap b \mathcal{A}$, and one may then apply Ehrhart theory to prove (generalizations of) Armstrong's conjecture.

3.2. Simultaneous Cores and Highest Weight Representations. As motivation for Theorem 1.1, we wish to show that the problem of computing the expected number of boxes in a simultaneous $(a, b)$-core is roughly equivalent to computing the expected norm of a weight in a particular highest weight representation. Having already related cores and $\Lambda^{\vee} \cap b \mathcal{A}$, we now wish to find a relation to representations.

In $\mathfrak{s l}_{a}$, there is a bijection between coweights inside the $b$-fold dilation of the fundamental alcove $b \mathcal{A}$, and coweights in the highest weight representation $\mathfrak{s l}_{a}\left(b \omega_{1}\right)$, where $\omega_{1}$ is the first fundamental weight — indeed, both are counted by the binomial coefficient $\left(\begin{array}{c}a+b \\ b\end{array}\right)$. This bijection may be described as follows, and is illustrated in Figure 4. We first center $b \mathcal{A}$ around the origin by sending

$$
x \mapsto x-\frac{b \rho^{\vee}}{h} .
$$

There is a natural bijection between the coweight and coroot lattice defined by

$$
\begin{aligned}
\Lambda^{\vee} & \rightarrow Q^{\vee} \\
x & \mapsto(1-c) x,
\end{aligned}
$$

where $c=(a, a-1, \ldots, 1) \in \mathfrak{S}_{a}$ is a long cycle. This reflects the fact that $(1-c)$ is conjugate to the Cartan matrix, with determinant equal to the index of connection $\left|Q^{\vee} / \Lambda^{\vee}\right|$.

Proposition 3.3. The composition of these two maps gives the desired bijection

$$
\begin{aligned}
\phi: \Lambda^{\vee} \cap b \mathcal{A} & \rightarrow \mathfrak{s l}_{a}\left(b \omega_{1}\right) \\
x & \mapsto(1-c)\left(x-\frac{b \rho^{\vee}}{h}\right) .
\end{aligned}
$$

Proof. The vertices of the polytope $b \mathcal{A}$ are $\{0\} \cup\left\{b \omega_{i}\right\}_{i=1}^{n-1}$, and $\Lambda^{\vee} \cap b \mathcal{A}$ is (by definition) all coweight points inside the convex hull of those vertices. On the other hand, the coweights in $\mathfrak{s l}_{a}\left(b \omega_{1}\right)$ are exactly those coweights in the convex hull of $\left\{c^{i}\left(b \omega_{1}\right)\right\}_{i=0}^{n-1}$ whose difference from $b \omega_{1}$ is in the coroot lattice. It therefore suffices to check that the map $(1-c)\left(x-\frac{b \rho^{\vee}}{h}\right)$ takes the vertices $\{0\} \cup\left\{\omega_{i}\right\}_{i=1}^{n-1}$ to the vertices $\left\{c^{i}\left(b \omega_{1}\right)\right\}_{i=0}^{n-1}$. But this is a simple computation-writing $e_{i}$ for the usual 
basis of $\mathbb{R}^{n}$ so that $\omega_{i}=\sum_{j=1}^{i} e_{j}-\frac{i}{n} \sum_{j=1}^{n} e_{j}$ and $\rho^{\vee}=\sum_{i=1}^{n-1} \omega_{i}$, we check

$$
\begin{aligned}
(1-c)\left(b \omega_{i}-\frac{b \rho^{\vee}}{h}\right) & =\frac{b(1-c)}{n}\left(\sum_{j=1}^{i}\left(\frac{n-1}{2}-i+j\right) e_{j}-\sum_{j=i+1}^{n}\left(\frac{n+1}{2}+i-j\right) e_{j}\right) \\
& =\frac{b}{n}\left((n-1) e_{i}+\sum_{j \neq i}-e_{j}\right)=c^{i}\left(b \omega_{1}\right) .
\end{aligned}
$$

3.3. Equivalence of Theorem 3.1 and Theorem 1.1 for $\mathfrak{s l}_{a}\left(b \omega_{1}\right)$. Under the bijection of Proposition 3.3, we show that computing the expected size $_{b}$ on $b \mathcal{A}$ (and hence the expected number of boxes in an $(a, b)$-core) is roughly equivalent to computing the expected norm on $\mathfrak{s l}_{a}\left(b \omega_{1}\right)$. Write $x_{b}=x-b \frac{\rho}{h}$ and compute:

$$
\begin{aligned}
\underset{\mu \in \mathfrak{s l}_{a}\left(b \omega_{1}\right)}{\mathbb{E}}(\langle\mu, \mu\rangle) & =\frac{1}{\left(\begin{array}{c}
a+b \\
b
\end{array}\right)} \sum_{\mu \in \mathfrak{s l}_{a}\left(b \omega_{1}\right)}\|x\|^{2}=\frac{1}{\left(\begin{array}{c}
a+b \\
b
\end{array}\right)} \sum_{\mu \in b \mathcal{A}}\left\|(1-c) x_{b}\right\|^{2} \\
& =\frac{1}{\left(\begin{array}{c}
a+b \\
b
\end{array}\right)} \sum_{\mu \in b \mathcal{A}}\left(2\left\|x_{b}\right\|^{2}-2\left\langle c x_{b}, x_{b}\right\rangle\right) \\
& =\frac{a^{2}-1}{6 a}+\frac{1}{\left(\begin{array}{c}
a+b \\
b
\end{array}\right)}\left(\frac{4}{a} \sum_{\mu \in b \mathcal{A}} \operatorname{size}_{b}(x)-2 \sum_{\mu \in b \mathcal{A}}\left\langle c x_{b}, x_{b}\right\rangle\right)
\end{aligned}
$$

It is slightly surprising, but follows from an Ehrhart-theoretic computation of roughly the same degree of difficulty as the computation of the expectation of size (the only difficulty arising from a repeated root), that

$$
\frac{1}{\left(\begin{array}{c}
a+b \\
b
\end{array}\right)} \sum_{\mu \in b \mathcal{A}}\left\langle c x_{b}, x_{b}\right\rangle=\frac{(a-5)(a-1) b(a+b)}{12 a(a+1)}
$$

so that

$$
\begin{aligned}
\underset{\mu \in \mathfrak{s}_{a}\left(b \omega_{1}\right)}{\mathbb{E}}(\langle\mu, \mu\rangle) & =\frac{a^{2}-1}{6 a}+\frac{4}{a} \frac{(a-1)(b-1)(a+b+1)}{24}-2 \frac{(a-5)(a-1) b(a+b)}{12 a(a+1)} \\
& =\frac{(a-1) b(a+b)}{a(a+1)} .
\end{aligned}
$$

On the other hand - since the representation is multiplicity-free - we could have applied Ehrhart theory directly to compute the expected norm of a weight in $\mathfrak{s l}_{a}\left(b \omega_{1}\right)$. We conclude that computing $\underset{\mu \in \mathfrak{s l}_{a}\left(b \omega_{1}\right)}{\mathbb{E}}(\langle\mu, \mu\rangle)$ is roughly equivalent to computing the expectation of size on simultaneous $(a, b)$-cores.

Thus, given the success of studying moments of norms of weights in $b \mathcal{A}$, we found it a reasonable extension to ask for the expected norm of a weight in a highest weight representation.

\section{Proof of Theorem 1.1 using the Weyl Character Formula}

In this section we use the polynomial method and the Weyl character formula (Theorem 2.1) to give an elementary, uniform proof of Theorem 1.1 in all types. We first prove polynomiality of sums of polynomial functions in the weights in $V_{\lambda}$. 


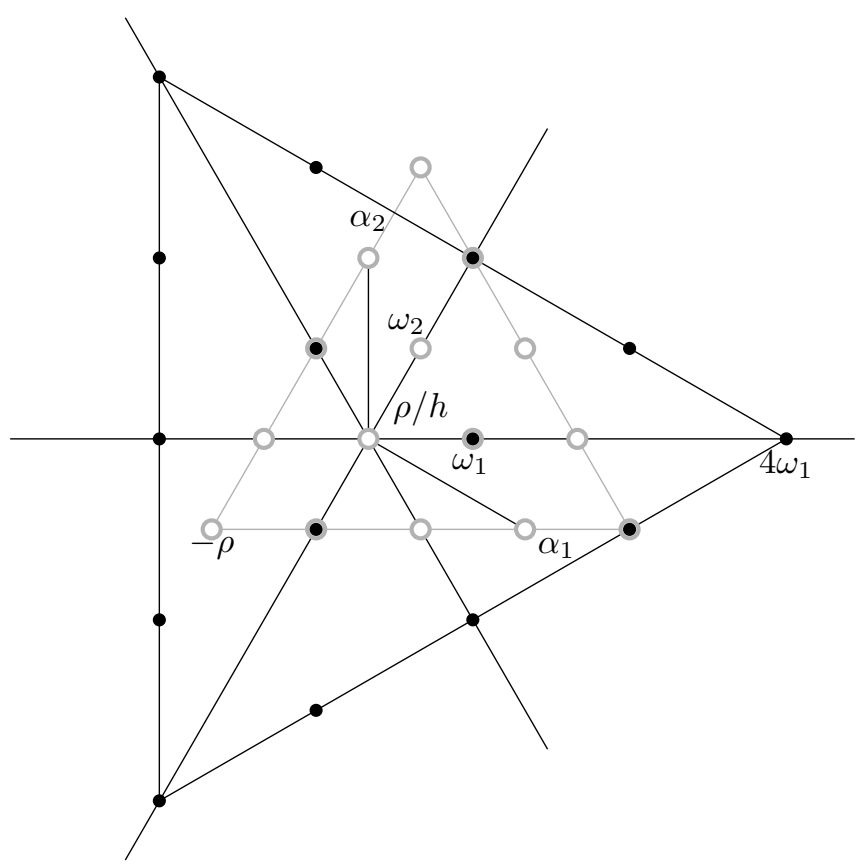

FiguRE 4. The weights inside a 4-fold dilation of the fundamental alcove in $\mathfrak{s l}_{3}$ are drawn as gray circles inside the gray triangle, while the weights in the representation $V_{4 \omega_{1}}$ are drawn as black disks. The statistic size on the weights in the dilation of the fundamental alcove is a quadratic form that is a slight modification of the norm.

Theorem 4.1. Suppose that $\lambda$ is a dominant integral weight of $\mathfrak{h}$ and let $V_{\lambda}$ be the finite dimensional irreducible representation of $\mathfrak{g}$ of highest weight $\lambda$. For a weight $\mu$, let $\operatorname{dim}\left(V_{\lambda}(\mu)\right)$ be the multiplicity of $\mu$ in $V_{\lambda}$. Let $P \in S(\mathfrak{h})^{W}$ be a $W$-invariant polynomial on $\mathfrak{h}^{*}$ of degree $d$. Then

$$
S(P, \lambda):=\frac{1}{\operatorname{dim}\left(V_{\lambda}\right)} \sum_{\mu \in \mathfrak{h}^{*}} \operatorname{dim}\left(V_{\lambda}(\mu)\right) P(\mu)
$$

is a polynomial in $\lambda$ of at most degree $d$. It is a $W$-invariant polynomial in variables given by $\lambda+\rho$.

Proof. We follow the ideas and notation of the derivation of the Weyl dimension formula from the Weyl character formula in [GW09, Section 7.1.2].

We write $f_{\lambda+\rho}=\frac{A_{\lambda+\rho}}{A_{\rho}}$. We define the linear differential operator

$$
\begin{aligned}
N: \mathcal{A}\left[\mathfrak{h}^{*}\right] & \rightarrow \mathcal{A}\left[\mathfrak{h}^{*}\right] \\
N\left(e^{\lambda}\right) & =P(\lambda) e^{\lambda},
\end{aligned}
$$

and the linear evaluation map

$$
\begin{aligned}
\epsilon: \mathcal{A}\left[\mathfrak{h}^{*}\right] & \rightarrow \mathbb{C} \\
\epsilon\left(e^{\lambda}\right) & =1 .
\end{aligned}
$$

Then we have that

$$
\frac{\epsilon\left(N\left(f_{\lambda+\rho}\right)\right)}{\epsilon\left(f_{\lambda+\rho}\right)}=\frac{\sum_{\mu \in \mathfrak{h}^{*}} \operatorname{dim}\left(V_{\lambda}(\mu)\right) P(\mu)}{\sum_{\mu \in \mathfrak{h}^{*}} \operatorname{dim}\left(V_{\lambda}(\mu)\right)}=S(P, \lambda) .
$$


Write $A_{\rho}=\prod_{\alpha \in \Phi^{+}}\left(e^{\alpha / 2}-e^{-\alpha / 2}\right)$. Now $N$ is a differential operator of degree $d$, so the quotient rule implies that

$$
\begin{aligned}
N\left(f_{\lambda}\right) & =N\left(\frac{A_{\lambda}}{A_{\rho}}\right)=N\left(\frac{A_{\lambda}}{\prod_{\alpha \in \Phi^{+}}\left(e^{\alpha / 2}-e^{-\alpha / 2}\right)}\right) \\
& =\sum \frac{B_{\lambda, \mathbf{m}}}{\prod_{\alpha \in \Phi^{+}}\left(e^{\alpha / 2}-e^{-\alpha / 2}\right)^{m_{\alpha}+1}}
\end{aligned}
$$

where the sum is over all $\mathbf{m}=\left(m_{\alpha}\right) \in[d]^{\Phi^{+}}$such that $\sum_{\alpha \in \Phi^{+}} m_{\alpha} \leq d$, and where $B_{\lambda, \mathbf{m}} \in \mathcal{A}\left[\mathfrak{h}^{*}\right]$ has coefficients that are polynomials in $\lambda$ of degree at most $d-\sum_{\alpha \in \Phi^{+}} m_{\alpha}$. Using L'Hôpital's rule we see that $\epsilon\left(N\left(f_{\lambda}\right)\right)$ is a polynomial in $\lambda$ of degree at most $\left|\Phi^{+}\right|+d$. It is alternating in $\lambda$. In particular, $\epsilon\left(N\left(f_{\lambda}\right)\right)=0$ if $\lambda$ is not regular. So for every $\alpha \in \Phi^{+}$the linear factor $\langle\lambda, \alpha\rangle$ divides the polynomial $\epsilon\left(N\left(f_{\lambda}\right)\right)$.

Consider the special case where $P=1$, so that $N$ is the identity and $d=0$. Then this implies that $\epsilon\left(N\left(f_{\lambda}\right)\right)=\epsilon\left(f_{\lambda}\right)=C \prod_{\alpha \in \Phi^{+}}\langle\lambda, \alpha\rangle$ for a constant $C$. We have that $f_{\rho}=e^{0}$ so that $C=\frac{1}{\prod_{\alpha \in \Phi^{+}}\langle\rho, \alpha\rangle}$.

For any $W$-invariant polynomial $P \in S(\mathfrak{h})^{W}$, the polynomial $\epsilon\left(f_{\lambda}\right)$ therefore divides the polynomial $\epsilon\left(N\left(f_{\lambda}\right)\right)$, so that the quotient $\frac{\epsilon\left(N\left(f_{\lambda}\right)\right)}{\epsilon\left(f_{\lambda}\right)}$ is a polynomial in $\lambda$ of degree at most $d$. It is $W$-invariant, since both $\epsilon\left(N\left(f_{\lambda}\right)\right)$ and $\epsilon\left(f_{\lambda}\right)$ are alternating in $\lambda$. Thus $S(\lambda, P)=\frac{\epsilon\left(N\left(f_{\lambda+\rho}\right)\right)}{\epsilon\left(f_{\lambda+\rho}\right)}$ is given by a $W$-invariant polynomial of degree at most $d$ in $\lambda+\rho$.

Example 4.2. Consider $\mathfrak{g}=\mathfrak{s l}_{2}$ and $P=\|\cdot\|^{2}$, where $\|\cdot\|^{2}$ is given by the $\mathfrak{S}_{2^{-}}$ equivariant polynomial $\|(x,-x)\|^{2}=2 x^{2}$. Since the dominant weights are given by $\lambda=(m,-m)$ for $m \in \mathbb{N}$, and since the weights in $V_{\lambda}$ are exactly of the form $(m,-m),(m-2,-m+2), \ldots,(-m, m)$ (with no multiplicity), we compute that

$$
\begin{aligned}
S\left(\|\cdot\|^{2}, \lambda\right)=\frac{2}{m+1} \sum_{i=0}^{m}(m-2 i)^{2}=\frac{2}{3} m(m+2) & =\frac{1}{3}\left(2(m+1)^{2}-2\right), \\
& =\frac{1}{h+1}\left(\|\lambda+\rho\|^{2}-\|\rho\|^{2}\right),
\end{aligned}
$$

which is $\mathfrak{S}_{2}$-equivariant as a polynomial in $m+1$.

First proof of Theorem 1.1. For this proof, we use the normalization of the Killing form that $\|\widetilde{\alpha}\|^{2}=\frac{1}{g}$, where $g$ is the dual Coxeter number. By Theorem $4.1, S(\|\cdot\|, \lambda)$ is a $W$-invariant polynomial of degree at most 2 in $\lambda+\rho$ so that $S\left(\|\cdot\|^{2}, \lambda\right)=$ $a+b\|\lambda+\rho\|^{2}$ for some $a, b \in \mathbb{C}$. We have that $S\left(\|\cdot\|^{2}, 0\right)=0$, so $a=-b\|\rho\|^{2}$. Furthermore, if $\widetilde{\alpha} \in \Phi^{+}$is the highest root, then $V_{\widetilde{\alpha}}$ is the adjoint representation of $\mathfrak{g}$, so we get

$$
S\left(\|\cdot\|^{2}, \widetilde{\alpha}\right)=\frac{1}{n(h+1)} \sum_{\alpha \in \Phi}\|\alpha\|^{2}=\frac{1}{h+1}
$$

using $\sum_{\alpha \in \Phi}\|\alpha\|^{2}=n$ for the Killing form [Bro64]. So $\frac{1}{h+1}=b\left(\|\widetilde{\alpha}+\rho\|^{2}-\|\rho\|^{2}\right)=b$, using that $\|\widetilde{\alpha}+\rho\|^{2}-\|\rho\|^{2}$ is the Casimir eigenvalue on the adjoint representation and therefore equals 1 . We conclude that

$$
S\left(\|\cdot\|^{2}, \lambda\right)=\frac{1}{h+1}\left(\|\lambda+\rho\|^{2}-\|\rho\|^{2}\right)
$$

\section{A Combinatorial Proof in Type $A$}

In this section, we make the polynomiality argument of the previous section more concrete using the combinatorics of the representation theory of $\mathfrak{s l}_{n}$. Fix $\mathfrak{g}=\mathfrak{s l}_{n}$ 
and $\omega_{i}=\sum_{j=1}^{i} e_{i}$. In $\mathfrak{s l}_{n}$, dominant weights of $\mathfrak{h}$ may be parametrized as integer partitions

$$
\lambda=\left[\lambda_{1} \geq \lambda_{2} \geq \cdots \geq \lambda_{n}\right] \vdash m,
$$

where parts $\lambda_{i}$ may be equal to zero. Fix a highest weight $\lambda$, and write

$$
\overline{\lambda_{i}}=\lambda_{i}-\frac{|\lambda|}{n}, \bar{\lambda}=\left[\overline{\lambda_{1}} \geq \cdots \geq \overline{\lambda_{n}}\right] \text {, and } \rho=[n-1, n-2, \ldots, 1,0] .
$$

With these conventions, weights $\mu$ in the highest weight representation $\mathfrak{s l}_{n}(\lambda)$ may be thought of as certain points in $\mathbb{R}^{n}$ with positive entries and sum equal to $m$. Combinatorially, the multiplicity of $\mu$ in $\mathfrak{s l}_{n}(\lambda)$ is well-known to be given by the number of semistandard tableaux of shape $\lambda$ on the alphabet $[n]$ with content $\mu ; m$ is just the number of boxes in the Ferrers shape $\lambda$ :

$$
\operatorname{ch}\left(\mathfrak{s l}_{n, \lambda}\right)=s_{\lambda}\left(x_{1}, \ldots, x_{n}\right)=\sum_{\substack{\text { semistandard } \\ \text { of shape } \lambda}} \mathbf{x}^{T}
$$

where $\mathbf{x}^{T}=\prod_{i=1}^{n} x_{i}^{|\{i \in T\}|}$ and $s_{\lambda}\left(x_{1}, \ldots, x_{n}\right)$ is a Schur polynomial. As a simple consequence of this combinatorial description of the character, we have Weyl's "interlacing" multiplicity-free formula for the branching of the representation $\mathfrak{s l}_{n, \lambda}$ to $\mathfrak{s l}_{n-1}$ :

$$
\mathfrak{s l}_{n, \lambda}=\bigoplus_{\mu} \mathfrak{s l}_{n-1, \mu}, \text { where } \lambda_{1} \geq \mu_{1} \geq \lambda_{2} \geq \cdots \geq \mu_{n-1} \geq \lambda_{n}
$$

By symmetry of the Schur function, and since this branching rule exactly peels off the boxes containing the entry $n$ (corresponding to the value of the coordinate $x_{n}$ ), one could imagine using this formula to determine the norm by computing

$$
\sum_{\mu \in V_{\lambda}} \operatorname{dim}\left(\mathfrak{s l}_{n, \lambda}(\mu)\right)\langle\mu, \mu\rangle=n \sum_{\mu} \operatorname{dim}\left(\mathfrak{s l}_{n-1, \mu}\right)(|\lambda|-|\mu|)^{2} .
$$

We do not follow this approach here, but instead isolate the boxes containing the entry $n$ using the Pieri rule and an inclusion-exclusion argument.

Theorem 5.1. Let $\mathfrak{g}=\mathfrak{s l}_{n}$. Suppose that $\lambda$ is a dominant weight of $\mathfrak{h}$ and let $\mathfrak{s l}_{n, \lambda}$ be the finite dimensional irreducible representation of $\mathfrak{s l}_{n}$ of highest weight $\lambda$. Then

$$
\frac{1}{\operatorname{dim}\left(\mathfrak{s l}_{n, \lambda}\right)} \sum_{\mu \in \mathfrak{s l}_{n}(\lambda)} \operatorname{dim}\left(\mathfrak{s l}_{n, \lambda}(\mu)\right)\langle\bar{\mu}, \bar{\mu}\rangle=\frac{1}{h+1}\langle\bar{\lambda}, \overline{\lambda+2 \rho}\rangle .
$$

Second proof of Theorem 1.1, valid in type A. Some care is needed when we compute the length of $\mu \in \mathfrak{s l}_{n}(\lambda)$-we wish to compute the length of the normalized weight $\bar{\mu}$. Of course, there is a simple relationship between the length of $\mu$ and of $\bar{\mu}:\langle\bar{\mu}, \bar{\mu}\rangle=\langle\mu, \mu\rangle-\frac{m^{2}}{n}$, where $m=\left\langle\mu,[1]^{n}\right\rangle$ (constant for all $\left.\mu \in \mathfrak{s l}_{n}(\lambda)\right)$. We may therefore compute with unnormalized weights using the relationship

$$
\begin{aligned}
\frac{1}{\operatorname{dim}\left(\mathfrak{s l}_{n, \lambda}\right)} \sum_{\mu \in \mathfrak{s l} n, \lambda} \operatorname{dim}\left(\mathfrak{s l}_{n, \lambda}(\mu)\right)\langle\bar{\mu}, \bar{\mu}\rangle= & -\frac{m^{2}}{n} \\
& +\frac{1}{\operatorname{dim}\left(\mathfrak{s l}_{n, \lambda}\right)} \sum_{\mu \in \mathfrak{s l}_{n, \lambda}} \operatorname{dim}\left(\mathfrak{s l}_{n, \lambda}(\mu)\right)\langle\mu, \mu\rangle .
\end{aligned}
$$

Define $n$ new partitions

$$
\lambda^{(i)}=\left[\lambda_{1}+1 \geq \lambda_{2}+1 \geq \cdots \geq \lambda_{i-1}+1 \geq \lambda_{i+1} \geq \cdots \geq \lambda_{n}\right] \text { for } 1 \leq i \leq n .
$$

Using the fact that Schur polynomials in the variables $x_{i}$ are symmetric, conditioning on which boxes of $\lambda$ contain the entry $n$, and using the Pieri rule allows us to write 


$$
\begin{aligned}
& \frac{1}{\operatorname{dim}\left(\mathfrak{s l}_{n, \lambda}\right)} \sum_{\mu \in \mathfrak{s l}_{n, \lambda}} \operatorname{dim}\left(\mathfrak{s l}_{n, \lambda}(\mu)\right)\langle\bar{\mu}, \bar{\mu}\rangle=-\frac{m^{2}}{n}+ \\
& \begin{array}{c}
\text { Schur polynomial } \\
\text { symmetry }
\end{array} \text { inclusion- } \\
& +\overbrace{\frac{n}{\operatorname{dim}\left(\mathfrak{s l}_{n, \lambda}\right)}}^{\text {symmetry }} \sum_{j=1}^{n} \overbrace{(-1)^{j+1}}^{\begin{array}{c}
\text { inclusion- } \\
\text { exclusion }
\end{array}}(\underbrace{s_{\lambda^{(j)}}\left([1]^{n-1}\right) \sum_{i=0}^{\lambda_{j}-(j-1)} h_{i}\left([1]^{n-1}\right)}_{\text {Pieri rule; leftover boxes contain } n} \underbrace{\left(\lambda_{j}-(j-1)-i\right)^{2}}_{\begin{array}{c}
\text { contribution to norm } \\
\text { of boxes containing } n
\end{array}}),
\end{aligned}
$$

where the alternating sum reflects an inclusion-exclusion argument that removes the over-count of those partitions that aren't contained in $\lambda$. The point is that the numerator has now been expressed as a polynomial.

Example 5.2. As in Figure 1, let $\lambda=(2,1)$ and $n=3$. We consider all eight semistandard tableaux of shape $(2,1)$ with entries at most 3 :

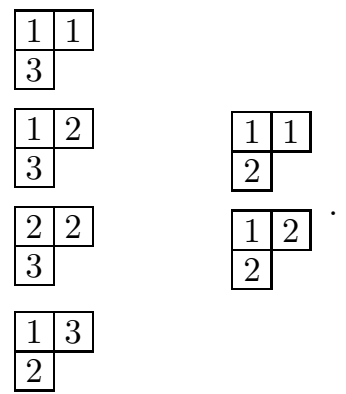

By symmetry of the Schur function,

$$
\sum_{T} \sum_{i=1}^{3}(\text { number of } i \text { in } \mathrm{T})^{2}=3 \sum_{T}(\text { number of } 3 \mathrm{~s} \text { in } \mathrm{T})^{2},
$$

and so we should group the tableaux by the number of boxes containing the entry $n=3$. The sum above uses the Pieri rule to do this, expressing these eight tableaux as

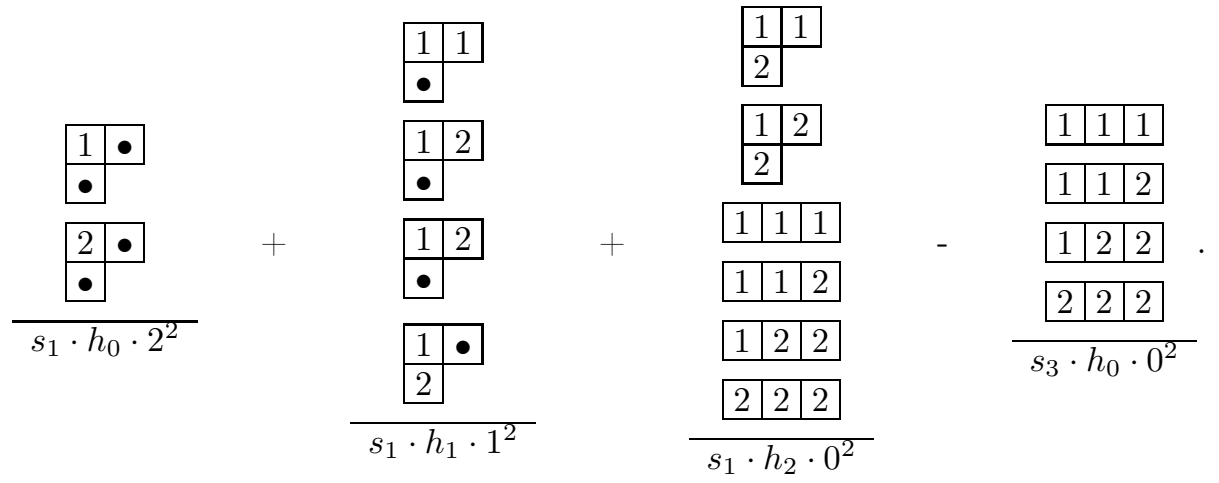

We have the evaluations of the Schur and homogeneous functions at $x_{i}=1$

$$
s_{\lambda}\left([1]^{n}\right)=\prod_{1 \leq i<j \leq n} \frac{\lambda_{i}-\lambda_{j}+j-i}{j-i} \text { and } h_{i}\left([1]^{n}\right)=\left(\begin{array}{c}
n+i-1 \\
i
\end{array}\right) .
$$

Dividing by $\operatorname{dim}\left(\mathfrak{s l}_{n}(\lambda)\right)=s_{\lambda}\left([1]^{n}\right)$, using the formulas above, performing the obvious cancellations, and explicitly evaluating the sums, we obtain 
(6) $\frac{1}{\operatorname{dim}\left(\mathfrak{s l}_{n, \lambda}\right)} \sum_{\mu \in \mathfrak{s l}_{n, \lambda}} \operatorname{dim}\left(\mathfrak{s l}_{n, \lambda}(\mu)\right)\langle\bar{\mu}, \bar{\mu}\rangle=$

$$
\begin{aligned}
& =-\frac{m^{2}}{n}+n ! \sum_{j=1}^{n}\left(\prod_{\substack{1 \leq i \leq n \\
i \neq j}} \frac{1}{\lambda_{j}-\lambda_{i}+i-j}\right)^{\lambda_{j}-(j-1)} \sum_{i=0}^{n+i-2}\left(\begin{array}{c}
n \\
i
\end{array}\right)\left(\lambda_{j}-(j-1)-i\right)^{2} \\
& =-\frac{m^{2}}{n}+\frac{n !}{n+1} \sum_{j=1}^{n}\left(\prod_{\substack{1 \leq i \leq n \\
i \neq j}} \frac{1}{\lambda_{j}-\lambda_{i}+i-j}\right)\left(\begin{array}{c}
n+\lambda_{j}-j \\
\lambda_{j}-j
\end{array}\right)\left(n+2\left(\lambda_{j}-j\right)+1\right) .
\end{aligned}
$$

On the other hand,

$$
\begin{aligned}
\frac{1}{h+1}\langle\bar{\lambda}, \overline{\lambda+2 \rho}\rangle & =\frac{1}{n+1}\left\langle\lambda-\frac{m}{n}[1]^{n}, \lambda-\frac{m}{n}[1]^{n}+2 \rho-(n-1)[1]^{n}\right\rangle \\
& =-\frac{m^{2}-m n+n^{2}}{n(n+1)}+\frac{1}{n+1}\left(\sum_{i=1}^{n} \lambda_{i}^{2}+2(n-i) \lambda_{i}\right)
\end{aligned}
$$

Setting Equations (6) and (8) equal, multiplying by $(n+1)$, pushing the constants to one side, and writing

$$
x_{j}=\lambda_{j}-j \text { and } P\left(x_{j}\right)=\left(n+2 x_{j}+1\right) \prod_{i=1}^{n}\left(x_{j}+i\right),
$$

we must check that

$$
\begin{array}{r}
2\left(\sum_{1 \leq i \leq j \leq n} x_{i} x_{j}\right)+(1+n)^{2}\left(\sum_{i=1}^{n} x_{i}\right)+\frac{n(n+1)\left(3 n^{2}+5 n+4\right)}{12} \\
=\sum_{j=1}^{n}\left(\prod_{\substack{\leq \leq i \leq n \\
i \neq j}} \frac{1}{x_{j}-x_{i}}\right) P\left(x_{j}\right) .
\end{array}
$$

Treating the $x_{i}$ as formal variables, Equation (9) is clearly a symmetric polynomial of degree 2 in the $x_{j}$. For any $1 \leq i<j \leq n$, the $i$ th and $j$ th terms of the sum in Equation (10) are the only two terms with $x_{i}-x_{j}$ in the denominator - and the sum of these terms is multiplied by $P\left(x_{j}\right)-P\left(x_{i}\right)$. This is true for any $i$ and $j$, and so all residues cancel. We conclude that since $P\left(x_{j}\right)$ has degree $n+1$, Equation (10) is also a symmetric polynomial in the $x_{j}$ of degree at most 2 . It remains to confirm that these are the same degree 2 symmetric function.

Setting $x_{i}=-i$ for $1 \leq i \leq n-2$, every term except the last two vanish in Equation (10). These remaining terms simplify to

$$
\begin{aligned}
& \left(\frac{n+2 x_{n}+1}{x_{n}-x_{n-1}} \frac{\prod_{i=1}^{n} x_{n}+i}{\prod_{i=1}^{n-2} x_{n}+i}\right)+\left(\frac{n+2 x_{n-1}+1}{x_{n-1}-x_{n}} \frac{\prod_{i=1}^{n} x_{n-1}+i}{\prod_{i=1}^{n-2} x_{n-1}+i}\right) \\
& =\frac{\left(n+2 x_{n}+1\right)\left(x_{n}+n-1\right)\left(x_{n}+n\right)-\left(n+2 x_{n-1}+1\right)\left(x_{n-1}+n-1\right)\left(x_{n-1}+n\right)}{x_{n}-x_{n-1}} \\
& =2\left(x_{n-1}^{2}+x_{n-2}^{2}\right)+2 x_{n} x_{n-1}+(5 n-1)\left(x_{n}+x_{n-1}\right)+4 n^{2}-n-1,
\end{aligned}
$$


which proves that the coefficients of $x_{i}^{2}$ and $x_{i} x_{j}$ in Equation (10) agree with those in Equation (9):

$$
\sum_{j=1}^{n}\left(\prod_{\substack{1 \leq i \leq n \\ i \neq j}} \frac{1}{x_{j}-x_{i}}\right) P\left(x_{j}\right)=2\left(\sum_{1 \leq i \leq j \leq n} x_{i} x_{j}\right)+C_{1}\left(\sum_{i=1}^{n} x_{i}\right)+C_{0} .
$$

We now determine $C_{1}$. Setting $x_{i}=-i$ for $1 \leq i \leq n$ makes every term in Equation (10) vanish; setting $x_{i}=-i+1$ leaves only the first term, which simplifies to $n(n+1)$. Specializing $x_{i}$ to these values in Equation (11) and subtracting, we obtain

$$
\begin{aligned}
& \left(\frac{n(n+1)(n+2)(3 n+1)}{12}-\frac{n(n+1)}{2} C_{1}+C_{0}\right) \\
& -\left(\frac{(n-1) n(n+1)(3 n-2)}{12}-\frac{(n-1) n}{2} C_{1}+C_{0}\right)=n^{3}+n^{2}-n C_{1} .
\end{aligned}
$$

Equating $n^{3}+n^{2}-n C_{1}=0-n(n+1)$, we obtain $C_{1}=(n+1)^{2}$.

Again setting $x_{i}=-i$ for $1 \leq i \leq n$ so that Equation (10) is 0 , we finally determine that $C_{0}=\frac{n(n+1)\left(3 n^{2}+5 n+4\right)}{12}$ by computing

$$
C_{0}=2\left(\sum_{1 \leq i \leq j \leq n} i j\right)-(n+1)^{2}\left(\sum_{i=1}^{n} i\right)=\frac{n(n+1)\left(3 n^{2}+5 n+4\right)}{12}
$$

\section{Proof of Theorem 1.1 using Orthogonal Decompositions}

In this section, we give a conceptual proof of Theorem 1.1 (in types other than $A$ and $C$ ) using the theory of orthogonal decompositions. Our strategy is to compute the trace of the degree two Casimir element $\Omega$ in two different ways on the representation $V_{\lambda}$.

6.1. Orthogonal Decompositions of Lie algebras. The usual decomposition of $\mathfrak{g}$ using a fixed Cartan subalgebra $\mathfrak{h}$ and the adjoint representation is given in Equation (2). Numerologically, this reflects the identity

$$
n(h+1)=n+n h=\operatorname{dim}(\mathfrak{h})+|\Phi| .
$$

But since $\operatorname{dim}(\mathfrak{h})=n$ divides $\operatorname{dim}(\mathfrak{g})=n(h+1)$, we might ask for a different decomposition of $\mathfrak{g}$ using a direct sum of $h+1$ Cartan subalgebras:

$$
\mathfrak{g}=\bigoplus_{i=0}^{h} \mathfrak{h}_{i}, \text { with } \mathfrak{h}_{i} \text { a Cartan subalgebra of } \mathfrak{g} \text { and } \mathfrak{h}_{0}=\mathfrak{h} .
$$

In fact, such a decomposition is always possible. More difficult is to require that these $h+1$ Cartan subalgebras are pairwise orthogonal with respect to the Killing form; such a decomposition is called an orthogonal decomposition. We refer the reader to [KKU81, KT94] for background and references, pausing only to remark that such decompositions have a number of applications, including Thompson's construction of his sporadic simple group from the Lie algebra of type $E_{8}$ [Tho76] and the construction of mutually unbiased bases for quantum cryptography [BSTW07].

We note that Kostant used a dual approach to the related numerological problem of trying to uniformly explain the duality between degrees and the heights of roots [Kos09]. Kostant decomposed $\mathfrak{g}$ into direct sum of $n$ irreducible representations of the principal three dimensional simple subalgebra (a distinguished copy of $\mathfrak{s l}_{2}$ inside $\left.\mathfrak{g}\right)$, reflecting the identity $n(h+1)=\sum_{i=1}^{n}\left(2 d_{i}-1\right)$. 
Theorem 6.1 ([KT94]). A complex simple Lie algebra $\mathfrak{g}$ has an orthogonal decomposition, except possibly if

- $\mathfrak{g}=\mathfrak{s l}_{n}$ for $n$ not a prime power; or if

- $\mathfrak{g}=\mathfrak{s p}_{2 n}$ for $n \neq 2^{m}$.

Although types $A$ and $C$ are usually the easiest Lie algebras to work with, it is widely believed that these classical Lie algebras do not have orthogonal decompositions (outside the cases listed above); this problem is wide open, even for $\mathfrak{s l}_{6}$.

The problem of finding such decompositions was dubbed the Winnie-the-Pooh problem in the Russian paper [KKU81], due to a play on words found in Zahoder's translation of Milne's famous children's book "Winnie-the-Pooh" into Russian. Zahoder's play on words can be interpreted as the sequence of Cartan types $A_{5}$ corresponding to the smallest open case $\mathfrak{s l}_{6}$ - then $A_{6}, A_{7}$, and $A_{8}$. This play on words apparently has no counterpart in Milne's original text, so when translating [KKU81] into English, Queen also translated Zahoder's verse - while managing to preserve the pun [KT94].

Third proof of Theorem 1.1, valid in types not $A$ or $C$. Suppose $\mathfrak{g}$ has an orthogonal decomposition $\mathfrak{g}=\bigoplus_{i=0}^{h} \mathfrak{h}_{i}$. For each $0 \leq i \leq h$, pick an orthonormal basis $\left\{X_{i, 1}, \ldots, X_{i, n}\right\}$ of $\mathfrak{h}_{i}$. Then

$$
\left\{X_{i, j}: 0 \leq i \leq h \text { and } 1 \leq j \leq n\right\}
$$

is an orthonormal basis of $\mathfrak{g}$, so we may write the degree two Casimir element $\Omega$ as

$$
\Omega=\sum_{\substack{0 \leq i \leq h \\ 1 \leq j \leq n}} X_{i, j}^{2}
$$

We compute the trace of $\Omega$ on $V_{\lambda}$ in two different ways. On the one hand, $\Omega$ acts as the scalar $\langle\lambda, \lambda+2 \rho\rangle$ by Theorem 2.2 , so that

$$
\operatorname{tr}_{V_{\lambda}}(\Omega)=\langle\lambda, \lambda+2 \rho\rangle \operatorname{dim}\left(V_{\lambda}\right) .
$$

On the other hand, for $0 \leq i \leq h$ define $\Omega_{i}=\sum_{j=0}^{n} X_{i, j}^{2}$. By definition, $X_{0, j}$ acts as $\mu\left(X_{0, j}\right)$ on the $\mu$-weight space of $V_{\lambda}$, so

$$
\operatorname{tr}_{V_{\lambda}}\left(\Omega_{0}\right)=\sum_{\mu \in V_{\lambda}} \operatorname{dim}\left(V_{\lambda}(\mu)\right) \sum_{j=1}^{n} \mu\left(X_{0, j}\right)^{2}=\sum_{\mu \in V_{\lambda}} \operatorname{dim}\left(V_{\lambda}(\mu)\right)\langle\mu, \mu\rangle,
$$

since $\left\{X_{0,1}, \ldots, X_{0, n}\right\}$ is an orthonormal basis of $\mathfrak{h}=\mathfrak{h}_{0}$. Since every $\mathfrak{h}_{i}$ is conjugate to $\mathfrak{h}_{0}$ under an inner automorphism of $\mathfrak{g}$ that leaves the Killing form invariant, we have that $\Omega_{i}$ is conjugate to $\Omega_{0}$ for all $0 \leq i \leq h$. Therefore,

$$
\begin{aligned}
\operatorname{tr}_{V_{\lambda}}(\Omega) & =\operatorname{tr}_{V_{\lambda}}\left(\sum_{i=0}^{h} \Omega_{i}\right)=\sum_{i=0}^{h} \operatorname{tr}_{V_{\lambda}}\left(\Omega_{i}\right) \\
& =(h+1) \operatorname{tr}_{V_{\lambda}}\left(\Omega_{0}\right)=(h+1) \sum_{\mu \in V_{\lambda}} \operatorname{dim}\left(V_{\lambda}(\mu)\right)\langle\mu, \mu\rangle .
\end{aligned}
$$

The result now follows from equating the two expressions for $\operatorname{tr}_{V_{\lambda}}(\Omega)$.

By Theorem 6.1, this proof of Theorem 1.1 applies to all types except possibly if $\mathfrak{g}=\mathfrak{s l}_{n}$ for $n$ composite; or if $\mathfrak{g}=\mathfrak{s p}_{2 n}$ for $n \neq 2^{m}$. 


\section{Coxeter Cumulants}

Let $[n]_{q}:=1+q+\cdots+q^{n-1}$ be the uniform distribution on $\{0,1, \ldots, n-1\}$.

Lemma 7.1. Fix $\left\{a_{i}\right\}_{i=1}^{k}$ and $\left\{b_{i}\right\}_{i=1}^{k}$ two sets of positive integers with $\prod_{i=1}^{k} \frac{\left[a_{i}\right]_{q}}{\left[b_{i}\right]_{q}}$ a polynomial in $q$, and let $X$ be a random variable with this distribution. Then the rth cumulant of $X$ is

$$
\kappa_{r}(X)=\frac{B_{r}}{r} \sum_{i=1}^{n}\left(a_{i}^{r}-b_{i}^{r}\right),
$$

where $B_{r}$ is the rth Bernoulli number. In particular, $\kappa_{r}(X)=0$ for odd $r>1$.

Proof. We first claim that the $r$ th cumulant of $[n]_{q}$ is

$$
\kappa_{r}\left([n]_{q}\right)=\frac{B_{r}}{r}\left(n^{r}-1\right) .
$$

Let $X$ be a random variable whose distribution is $[n]_{q}$, and let

$$
u(t)=\ln \mathbb{E}\left(e^{t X}\right)=\sum_{r=0}^{\infty} \kappa_{r}(X) \frac{t^{r}}{r !}
$$

be its cumulant exponential generating function. We claim that

$$
u(t)=\ln (n)+\sum_{r=1}^{\infty} \frac{B_{r}}{r}\left(n^{r}-1\right) \frac{t^{r}}{r},
$$

so that the coefficients of $\frac{t^{r}}{r !}$ are as desired. To prove Equation (14), we write

$$
u(t)=\ln \left(\sum_{i=0}^{n-1} e^{i t}\right)=\ln \left(\frac{1-e^{n t}}{1-e^{t}}\right) .
$$

Taking a derivative of Equation (14) using the fact that $\sum_{r=0}^{\infty} B_{r} \frac{t^{r}}{r !}=\frac{t}{1-e^{-t}}$, we obtain

$$
\sum_{r=1}^{\infty} B_{r}\left(n^{r}-1\right) \frac{t^{r}-1}{r !}=\frac{1}{t}\left(\frac{n t}{1-e^{-n t}}-\frac{t}{1-e^{-t}}\right)
$$

which matches the derivative $u^{\prime}(t)$. The constant term is verified by computing $\lim _{t \rightarrow 0} u(t)$.

Suppose now $X$ is a random variable with distribution $\prod_{i=1}^{k} \frac{\left[a_{i}\right]_{q}}{\left[b_{i}\right]_{q}}$. Using Equation (13), we compute

$$
\kappa_{r}(X)=\kappa_{r}\left(\prod_{i=1}^{k} \frac{\left[a_{i}\right]_{q}}{\left[b_{i}\right]_{q}}\right)=\sum_{i=1}^{k} \kappa_{r}\left(\left[a_{i}\right]_{q}\right)-\kappa_{r}\left(\left[b_{i}\right]_{q}\right)=\frac{B_{r}}{r} \sum_{i=1}^{k}\left(a_{i}^{r}-b_{i}^{r}\right) .
$$

The remainder of this section is devoted to corollaries of Lemma 7.1.

7.1. Coxeter numerology. A finite irreducible Coxeter group $W$ of rank $n$ has roots $\Phi$, positive roots $\Phi^{+}$, degrees $d_{1}<\cdots<d_{n}$, exponents $e_{1}<\cdots<e_{n}$, and a Coxeter number $h$. These satisfy $e_{i}=d_{i}-1, h=d_{n}$, and

$$
\sum_{i=1}^{n} e_{i}=\frac{n h}{2}=\left|\Phi^{+}\right| .
$$

Following [BS12, Sut98a], for $W$ a finite Weyl group (a crystallographic Coxeter group), define

$$
\gamma=\frac{\langle\widetilde{\alpha}, \widetilde{\alpha}\rangle}{\left\langle\widetilde{\alpha}_{s}, \widetilde{\alpha}_{s}\right\rangle} g g^{\vee}
$$

where $\widetilde{\alpha}$ is the highest root, $\widetilde{\alpha}_{s}$ is the highest short root, $g$ is the dual Coxeter number of $\Phi$, and $g^{\vee}$ is the dual Coxeter number of the dual root system $\Phi^{\vee}$. For 
noncrystallographic types, $\gamma$ is defined in Figure 5. With this definition, R. Suter found the striking uniform formulas [Sut98a]

$$
\sum_{i=1}^{n} e_{i}^{2}=\frac{n\left(h^{2}+\gamma-h\right)}{6} \text { and } \sum_{i=1}^{n} e_{i}^{3}=\frac{n h(\gamma-h)}{4} .
$$

\begin{tabular}{|c|cccccccc|}
\hline Type & $A_{n}$ & $B_{n} / C_{n}$ & $D_{n}$ & $E_{6}$ & $E_{7}$ & $E_{8}$ & $F_{4}$ & $G_{2}$ \\
$\gamma$ & $(n+1)^{2}$ & $4 n^{2}+2 n-2$ & $(2 n-2)^{2}$ & 144 & 324 & 900 & 162 & 48 \\
\hline
\end{tabular}

\begin{tabular}{|c|ccc|}
\hline Type & $H_{3}$ & $H_{4}$ & $I_{2}(m)$ \\
$\gamma$ & 124 & 1116 & $2 m^{2}-5 m+6$ \\
\hline
\end{tabular}

Figure 5. Values of $\gamma$ for finite Coxeter groups. The definition of $\gamma$ is uniform for Weyl groups.

7.2. Inversions in Finite Coxeter Groups. Let $(W, S)$ be a finite irreducible Coxeter system. The length of the shortest word in simple reflections for an element $w \in W$ is written $\ell(w)$. It is well-known that the generating function for length is

$$
\operatorname{Inv}(W ; q):=\sum_{w \in W} q^{\ell(w)}=\prod_{i=1}^{n}\left[d_{i}\right]_{q} .
$$

Applying Lemma 7.1 gives the following generalization of [KS18, Theorem 3.1].

Corollary 7.2. Let $W$ be a finite irreducible Coxeter group, and let $X$ be a random variable with distribution $\operatorname{Inv}(W ; q)$. Then

$$
\kappa_{r}(X)=\frac{B_{r}}{r} \sum_{i=1}^{n}\left(d_{i}^{r}-1\right) .
$$

In particular, the expectation and variance are

$$
\mathbb{E}(X)=\frac{\left|\Phi^{+}\right|}{2} \text { and } \mathbb{V}(X)=\frac{n\left(\gamma+5 h+h^{2}\right)}{72} .
$$

Proof. The formula for expectation is immediate from the symmetry $w \mapsto w w_{\circ}$, where $w_{\circ}$ is the longest element of $W$. The formula for variance follows from Equation (15).

7.3. Rational Catalan Numbers. Let $W$ be a finite well-generated irreducible complex reflection group with exponents $e_{1}, \ldots, e_{n}$ and $p$ coprime to to the Coxeter number $h=d_{n}$. The rational Catalan number

$$
\mathrm{Cat}^{[p]}(W ; q):=\prod_{i=1}^{n} \frac{\left[p+\left(p e_{i} \bmod h\right)\right]_{q}}{\left[d_{i}\right]_{q}}
$$

is the graded character of the representation $e L_{p / h}$ (triv) of the rational Cherednik algebra corresponding to $W$ [GG12, STW18]. When $W$ is a Coxeter group, multiplication by $p$ simply permutes the exponents modulo $h$. Applying Lemma 7.1 gives the following.

Corollary 7.3. Let $W$ be a finite Coxeter group, and let $X$ be a random variable with distribution $\mathrm{Cat}^{[p]}(W ; q)$. Then

$$
\mathbb{E}(X)=\frac{n(p-1)}{2}, \quad \mathbb{V}(X)=\frac{n(p-1)(p+h+1)}{12},
$$

and for $W$ a crystallographic Coxeter group $\kappa_{4}(X)=-\frac{n(p-1)(h+p+1)\left(p^{2}+p h+\gamma+1\right)}{120}$. 
Proof. The formulas follow from simple computations and Equation (15).

Interestingly, the variance in Corollary 7.3 is exactly the expectation computed in [TW17] for simply-laced crystallographic Coxeter groups.

7.4. Minuscule Posets. Recall that a dominant weight $\lambda$ is called minuscule if the weights in its highest weight representation $V_{\lambda}$ coincides with weights in its Weyl group orbit $\{w \lambda: w \in W\}$. A minuscule poset may then be defined as the order filter of positive roots generated by simple root whose corresponding fundamental weight is minuscule.

A plane partition of height at most $k$ in a poset $P$ is an order-preserving map $P \rightarrow\{0,1, \ldots, k\}$. It is well-known that the generating function for the number of boxes in plane partitions of height at most $k$ in a minuscule poset has the product formula [Pro84]

$$
\operatorname{PP}^{[p]}(P ; q):=\prod_{p \in P} \frac{[k+\operatorname{ht}(p)]_{q}}{[\operatorname{ht}(p)]_{q}} .
$$

Corollary 7.4. Let $P$ be a minuscule poset, and let $X$ be a random variable with distribution $\mathrm{PP}^{[p]}(P ; q)$. Then

$$
\mathbb{E}(X)=\frac{k}{2}|P| \text { and } \mathbb{V}(X)=\frac{k(k+h)}{12}|P| .
$$

Proof. We have that

$$
\kappa_{r}(X)=\frac{B_{r}}{r} \sum_{p \in P}\left((k+\mathrm{ht}(p))^{r}-\mathrm{ht}(p)^{r}\right) .
$$

Expectation is immediate. For variance, we compute

$$
\begin{aligned}
\frac{1}{12} \sum_{p \in P}\left((k+\mathrm{ht}(p))^{2}-\mathrm{ht}(p)^{2}\right) & =\frac{1}{12} \sum_{p \in P}\left(k^{2}+2 k \mathrm{ht}(p)\right) \\
& =\frac{1}{12}\left(|P| k^{2}+2 k \sum_{p \in P} \mathrm{ht}(\mathrm{p})\right) \\
& =\frac{1}{12}\left(|P| k^{2}+|P| h k\right)=\frac{k(k+h)}{12}|P|,
\end{aligned}
$$

where $\sum_{p \in P} \operatorname{ht}(\mathrm{p})=\frac{h}{2}|P|$ using the symmetry of $P$.

Specializing to type $A$ gives the following.

Corollary 7.5. The variance for the number of boxes in plane partitions fitting inside an $a \times b \times c$ box is $\frac{1}{12} a b c(a+b+c)$.

A linear extension of a poset $P$ is an order-preserving bijection from $P$ to $\{1,2, \ldots,|P|\}$. Relative to a fixed linear extension $\ell$ of a poset, the major index of a second linear extension $\ell^{\prime}$ is the sum of the positions of the descents of $\ell^{\prime}$ - that is, the sum $\sum i$, where the sum is over all $i$ for which $\ell^{\prime}\left(\ell^{-1}(i)\right)>\ell^{\prime}\left(\ell^{-1}(i+1)\right)$. Recall that the generating function for major index of linear extensions of a minuscule poset is given by

$$
\operatorname{SYT}(P ; q):=\frac{[|P|] !_{q}}{\prod_{p \in P}[\mathrm{ht}(p)]_{q}} .
$$

Corollary 7.6. Let $P$ be a minuscule poset, and let $X$ be a random variable with distribution $\operatorname{SYT}(P ; q)$. Then $\mathbb{E}(X)=\frac{|P|(|P|+1-h)}{4}$. 
Proof. Compute using

$$
\kappa_{r}(X)=\frac{B_{r}}{r}\left(\sum_{i=1}^{|P|} i^{r}-\sum_{p \in P} \mathrm{ht}(p)^{r}\right) .
$$

Specializing to type $A$ gives the following.

Corollary 7.7. The expected value for major index of standard Young tableaux of rectangular shape is given by $\frac{a(a-1) b(b-1)}{4}$.

7.5. Descending Plane Partitions. The generating function for descending plane partitions by number of boxes is given by

$$
\operatorname{DPP}(q)=\prod_{i=0}^{n-1} \frac{[3 i+1] !_{q}}{[n+i] !_{q}} .
$$

Corollary 7.8. Let $X$ be a random variable with distribution $\operatorname{DPP}(q)$. Then

$$
\mathbb{E}(X)=\frac{1}{6} n\left(n^{2}-1\right) \text { and } \mathbb{V}(X)=\frac{1}{12} n^{2}\left(n^{2}-1\right) .
$$

\section{OPEN PROBLEMS}

- The problem of determining uniform formulas for higher moments for the expected norm of a weight in a highest weight representation is open.

- By the Harish-Chandra isomorphism, g has $n$ Casimir elements. Since the Casimir elements live in the center of $U(\mathfrak{g})$, they generalize the degree two Casimir by acting as a scalar on any highest weight representation $V(\lambda)$. It might be interesting to write down explicit formulas. ${ }^{1}$

- The expectations arising from evaluating other natural $W$-symmetric functions besides the norm on the weights of $V_{\lambda}$ could be worth looking at.

- Since Schubert polynomials generalize Schur polynomials - which are equivalent to the Weyl character formula in type $A$ - one could ask for expectation of polynomial functions of the exponents in a Schubert polynomial.

- For $P$ a minuscule poset, it would be interesting to find uniform expressions for $\sum_{p \in P}$ ht $(p)^{r}$.

\section{ACKNOWLEDGMENTS}

The second author warmly thanks Dennis Stanton for precious help with Section 5 and Paul Garrett for explaining to him where the Casimir element lives. An extended abstract of this work was presented at FPSAC 2018 [TW18].

\section{REFERENCES}

[Agg15] A. Aggarwal, Armstrong's conjecture for $(k, m k+1)$-core partitions, European Journal of Combinatorics 47 (2015), 54-67.

[AHJ14] D. Armstrong, C. Hanusa, and B. Jones, Results and conjectures on simultaneous core partitions, European Journal of Combinatorics 41 (2014), 205-220.

[And02] J. Anderson, Partitions which are simultaneously $t_{1}$-and $t_{2}$-core, Discrete Mathematics 248 (2002), no. 1, 237-243.

[Arm15] D. Armstrong, Rational Catalan combinatorics, 2012 (accessed 12 May, 2015).

[Bro64] Gordon Brown, A remark on semisimple Lie algebras, Proc. Amer. Math. Soc, vol. 15, 1964, p. 518.

\footnotetext{
${ }^{1}$ After our presentation of this work at FPSAC 2018, we understand that Richard Stanley has made some progress on this problem in type $A$.
} 
[BS12] J. Burns and R. Suter, Power sums of Coxeter exponents, Advances in Mathematics 231 (2012), no. 3-4, 1291-1307.

[BSTW07] P. Boykin, M. Sitharam, P. Tiep, and P. Wocjan, Mutually unbiased bases and orthogonal decompositions of Lie algebras, Quantum Information \& Computation 7 (2007), no. $4,371-382$.

[GG12] I. Gordon and S. Griffeth, Catalan numbers for complex reflection groups, American Journal of Mathematics 134 (2012), no. 6, 1491-1502.

[GW09] R. Goodman and N. Wallach, Symmetry, representations, and invariants, vol. 255, Springer, 2009.

[Hai94] M. Haiman, Conjectures on the quotient ring by diagonal invariants, Journal of Algebraic Combinatorics 3 (1994), no. 1, 17-76.

[Joh15] P. Johnson, Lattice points and simultaneous core partitions, 2015.

[KKU81] A. Kostrikin, I. Kostrikin, and V. Ufnarovskii, Orthogonal decompositions of simple Lie algebras (type $A_{n}$ ), Trudy Matematicheskogo Instituta imeni VA Steklova 158 (1981), 105-120.

[Kos09] B. Kostant, The principal three-dimensional subgroup and the betti numbers of a complex simple Lie group, Collected Papers (2009), 130-189.

[KS18] T. Kahle and C. Stump, Counting inversions and descents of random elements in finite Coxeter groups, arXiv preprint arXiv:1802.01389 (2018).

[KT94] A. Kostrikin and P. Tiep, Orthogonal decompositions and integral lattices, vol. 15, Walter de Gruyter, 1994.

[Pro84] R. Proctor, Bruhat lattices, plane partition generating functions, and minuscule representations, European Journal of Combinatorics 5 (1984), no. 4, 331-350.

[STW18] C. Stump, H. Thomas, and N. Williams, Cataland: why the fuss?, arXiv preprint arXiv:1503.00710 (2018).

[Sut98a] R. Suter, Coxeter and dual Coxeter numbers, Communications in Algebra 26 (1998), no. $1,147-153$.

[Sut98b] The number of lattice points in alcoves and the exponents of the finite Weyl groups, Mathematics of computation (1998), 751-758.

[SZ15] R. Stanley and F. Zanello, The catalan case of Armstrong's conjecture on simultaneous core partitions, SIAM Journal on Discrete Mathematics 29 (2015), no. 1, 658-666.

[Tho76] J. Thompson, A conjugacy theorem for $e_{8}$, Journal of Algebra 38 (1976), no. 2, 525530.

[TW17] M. Thiel and N. Williams, Strange expectations and simultaneous cores, Journal of Algebraic Combinatorics (2017), 1-43.

[TW18] — Winnie-the-pooh and the strange expectations, Discrete Mathematics and Theoretical Computer Science (2018).

(M. Thiel) Jane Street Capital, London, United Kingdom

E-mail address: thiel.marko@gmail.com

(N. Williams) University of Texas at Dallas, USA

E-mail address: nathan.f.williams@gmail.com 